\title{
EIGENVALUES OF COLLAPSING DOMAINS AND DRIFT LAPLACIANS
}

\author{
Zhiqin Lu AND Julie Rowlett
}

\begin{abstract}
By introducing a weight function to the Laplace operator, Bakry and Émery defined the "drift Laplacian" to study diffusion processes. Our first main result is that, given a Bakry-Émery manifold, there is a naturally associated family of graphs whose eigenvalues converge to the eigenvalues of the drift Laplacian as the graphs collapse to the manifold. Applications of this result include a new relationship between Dirichlet eigenvalues of domains in $\mathbb{R}^{n}$ and Neumann eigenvalues of domains in $\mathbb{R}^{n+1}$ and a new maximum principle. Using our main result and maximum principle, we are able to generalize all the results in Riemannian geometry based on gradient estimates to Bakry-Émery manifolds.
\end{abstract}

\section{Introduction}

Bakry-Émery geometry was introduced in [3] to study diffusion processes. For a Riemannian manifold $(M, g)$ and $\phi \in \mathcal{C}^{2}(M)$, the Bakry-Émery manifold is a triple $(M, g, \phi)$, where the measure on $M$ is the weighted measure $\mathrm{e}^{-\phi} d V_{g}$. If Ric and $\Delta$ are, respectively, the Ricci curvature and Laplacian with respect to the Riemannian metric $g$, then the Bakry-Émery Ricci curvature is defined to be ${ }^{1}$

$$
\operatorname{Ric}_{\infty}=\operatorname{Ric}+\operatorname{Hess}(\phi)
$$

and the Bakry-Émery Laplacian is

$$
\Delta_{\phi}=\Delta-\nabla \phi \cdot \nabla
$$

The operator can be extended as a self-adjoint operator with respect to the weighted measure $\mathrm{e}^{-\phi} d V_{g}$; it is also known as a "drifting" or "drift" Laplacian.

Theorem 1. Let $(M, g, \phi)$ be a compact Bakry-Émery manifold. Let

$$
M_{\varepsilon}:=\left\{(x, y) \mid x \in M, \quad 0 \leq y \leq \varepsilon \mathrm{e}^{-\phi(x)}\right\} \subset M \times \mathbb{R}^{+},
$$

with $\phi \in \mathcal{C}^{2}(M)$ and $\mathrm{e}^{-\phi} \in \mathcal{C}(M \cup \partial M)$. Let $\left\{\mu_{k}\right\}_{k=0}^{\infty}$ be the eigenvalues of the BakryÉmery Laplacian on $M$. If $\partial M \neq \emptyset$, assume the Neumann boundary condition. Let $\mu_{k}(\varepsilon)$ be the Neumann eigenvalues of $M_{\varepsilon}$ for $\tilde{\Delta}:=\Delta+\partial_{y}^{2}$. Then $\mu_{k}(\varepsilon)=\mu_{k}+O\left(\varepsilon^{2}\right)$ for $k \geq 0$.

A corollary of Theorem 1 gives a relationship between the Dirichlet eigenvalues in $\mathbb{R}^{n}$ and Neumann eigenvalues in $\mathbb{R}^{n+1}$.

Received by the editors August 18, 2011.

${ }^{1}$ In the notation of [18], this is the $\infty$ Bakry-Émery Ricci curvature; see also [24]. 
Corollary 1. Let $M$ be a bounded domain in $\mathbb{R}^{n}$ with smooth boundary, and let $\phi_{1}$ be the first Dirichlet eigenfunction of the Euclidean Laplacian on M. Define

$$
M_{\varepsilon}:=\left\{(x, y) \in \mathbb{R}^{n+1} \mid x \in M, 0 \leq y \leq \varepsilon \phi_{1}(x)^{2}\right\} .
$$

Let $\left\{\lambda_{k}\right\}_{k=1}^{\infty}$ be the Dirichlet eigenvalues of $M$, and let $\left\{\mu_{k}(\varepsilon)\right\}_{k=0}^{\infty}$ be the Neumann eigenvalues of $M_{\varepsilon}$. Then $\lim _{\varepsilon \rightarrow 0} \mu_{k-1}(\varepsilon)=\lambda_{k}-\lambda_{1}$, for all $k \in \mathbb{N}$.

In the second part of the paper, we establish a new maximum principle which, together with Theorem 1, imply the following.

Principle. There is a one-one correspondence between the gradient estimate on a Riemannian manifold and on a Barky-Émery manifold. More precisely, the eigenvalue estimate on the Bakry-Émery manidold $(M, g, \phi)$ is equivalent to that on the Riemannian manifold $\left(M_{\varepsilon}, g+d y^{2}\right)$ for $\varepsilon$ small enough.

The method of gradient estimates in eigenvalue problems was first used by $\mathrm{Li}-$ Yau [15]. The papers $[22,25,26,14,4,28,2,27]$ are the most influential to this work. Gradient estimates on Riemannian manifolds are often quite complicated. The point of the above Principle is that one may apply all the proofs of gradient estimates directly to Bakry-Émery geometry without repeating the calculations.

We end this section by discussing an alternative but related setting. ${ }^{2}$ In [18], Lott considered the product $M \times S^{q}$ for $q>0$, with warped product metric

$$
g_{i}^{M \times S^{q}}=g^{M}+\frac{1}{i^{2}} \phi^{\frac{2}{q}} g^{S^{q}} .
$$

A curvature bound of the type

$$
\operatorname{Ric}_{\phi}^{q}=\operatorname{Ric}^{M}+\operatorname{Hess}(\phi)-\frac{1}{q} d \phi \otimes d \phi \geq r g^{M}
$$

for some constant $r$ implies a lower bound of the curvature on $M \times S^{q}$ :

$$
\operatorname{Ric}^{M \times S^{q}} \geq r_{1} g^{M \times S^{q}}
$$

for a possibly smaller $r_{1}$, as $i \rightarrow \infty$. In this setting, the manifolds $\left(M_{i}, g_{i}\right)$ converge with respect to Gromov-Hausdorff norm to the manifold $\left(M, g^{M}\right)$. On the one hand, $q \in \mathbb{N}$ allows higher dimensional collapse, whereas we consider only one collapsing direction. On the other hand, the fibers in [18] are closed, which eliminates the need for estimates regarding the boundary of $M_{\varepsilon}$. In order to prove convergence of the eigenvalues in the setting of [18], one could apply Theorem 7.3 from Cheeger and Colding [6], but one must first prove that the gradient of the $k$ th eigenfunction is bounded above uniformly in $i$ (cf. equation (7.4) of [6]). Moreover, to prove the uniform eigenvalue convergence (for each $k$ ) as done here, one would need to demonstrate technical results analogous to those in Section 5. In the case of one collapsing direction, the $L^{2}$ bounds on the eigenfunctions are sufficient to prove uniform convergence of the eigenvalues. When more than one dimension collapses, some of the arguments in Section 3 will not apply. However, in the closed case, further techniques are available and boundary estimates are obsolete, so it may be possible to generalize our arguments. This will be carried out elsewhere.

The eigenvalues of the Laplacian on manifolds with boundary which collapse or are "very thin in one direction" has been studied by several authors. An estimate

\footnotetext{
${ }^{2}$ We are grateful to O. Munteanu for bringing this reference to our attention.
} 
and convergence result for the Dirichlet Laplacian on planar domains were obtained by [11] and later refined in [8]. In all dimensions, there is motivation from physics to understand the spectrum of the Laplacian for "thin tubes", see [10]. Our work can be seen as the higher dimensional Neumann analogue of [11] in the more general setting of Bakry-Émery geometry. It would be interesting to determine further terms in the expansion of $\mu_{k}(\varepsilon)$ as $\varepsilon \rightarrow 0$ in the spirit of Friedlander and Solomyak's work in two dimensions [8]. This investigation will be the subject of forthcoming work.

The organization of the paper is as follows. In Section 2, we present the variational principles for the drift Laplacian, which we use heavily in our proof. The proof of Theorem 1 and Corollary 1 comprise Section 3. We prove the new maximum principle and discuss its applications in Section 4; finally, Section 5 contains technical results on Schauder estimates which are of independent interest.

\section{Variational principles}

On a Riemannian manifold $(M, g)$ with boundary $\partial M$, the Laplace operator can be written as

$$
\Delta=\frac{1}{\sqrt{\operatorname{det}(g)}} \sum_{i, j} \partial_{i} g^{i j} \sqrt{\operatorname{det}(g)} \partial_{j}
$$

and in particular on $\mathbb{R}^{n}$ with the Euclidean metric,

$$
\Delta=\sum_{j=1}^{n} \frac{\partial^{2}}{\partial x_{j}^{2}}
$$

The Dirichlet (respectively, Neumann) eigenvalues of the Laplace operator are the real numbers $\lambda$ for which there exists an eigenfunction

$$
u \in \mathcal{C}^{\infty}(M) \text { such that }-\Delta u=\lambda u \text { and }\left.u\right|_{\partial M}=0,\left(\text { respectively, }\left.\frac{\partial u}{\partial n}\right|_{\partial M}=0\right) .
$$

The eigenvalues of the drift Laplace operator are defined analogously.

We shall use $\lambda$ to denote Dirichlet eigenvalues, $\mu$ to denote Neumann eigenvalues, and index the Dirichlet eigenvalues by $\mathbb{N}$ and the Neumann eigenvalues by $0 \cup \mathbb{N}$. The Dirichlet and Neumann ${ }^{3}$ eigenvalues, respectively, satisfy the following variational principles [5]:

$$
\begin{aligned}
& \lambda_{k}=\inf _{\varphi \in \mathcal{C}^{1}(M)}\left\{\frac{\int_{M}|\nabla \varphi|^{2}}{\int_{M} \varphi^{2}}|\varphi|_{\partial M}=0, \varphi \not \equiv 0=\int_{M} \varphi \phi_{l}, 0 \leq l<k\right\}, \\
& \mu_{j}=\inf _{\varphi \in \mathcal{C}^{1}(M)}\left\{\frac{\int_{M}|\nabla \varphi|^{2}}{\int_{M} \varphi^{2}} \mid \varphi \neq \equiv=\int_{M} \varphi \varphi_{l},-1 \leq l<j\right\},
\end{aligned}
$$

for $k \geq 1$ and $j \geq 0$, where $\phi_{j}$ and $\varphi_{l}$ are, respectively, eigenfunctions for $\lambda_{j}$ and $\mu_{l}$ (assuming that $\phi_{0} \equiv 0$ and $\varphi_{-1} \equiv 0$ ).

\footnotetext{
${ }^{3}$ Note that the Neumann boundary condition is automatically satisfied if no boundary condition is imposed in the variational principle.
} 
Proposition 1. Let $(M, g, \phi)$ be a Bakry-Émery manifold with boundary. Then, the Dirichlet and Neumann eigenvalues of the associated drift Laplacian satisfy the following variational principles:

$$
\begin{aligned}
& \lambda_{k}=\inf _{\varphi \in \mathcal{C}^{1}(M)}\left\{\frac{\int_{M}|\nabla \varphi|^{2} \mathrm{e}^{-\phi}}{\int_{M} \varphi^{2} \mathrm{e}^{-\phi}}|\varphi|_{\partial M}=0, \varphi \not \equiv 0=\int_{M} \varphi \phi_{j} \mathrm{e}^{-\phi}, 0 \leq j<k,\right\}, \\
& \mu_{j}=\inf _{\varphi \in \mathcal{C}^{1}(M)}\left\{\frac{\int_{M}|\nabla \varphi|^{2} \mathrm{e}^{-\phi}}{\int_{M} \varphi^{2} \mathrm{e}^{-\phi}} \mid \varphi \neq 0=\int_{M} \varphi \varphi_{l} \mathrm{e}^{-\phi},-1 \leq l<j\right\}
\end{aligned}
$$

for $k \geq 1$ and $j \geq 0$.

Remark 1. Let $\left\{\lambda_{k}\right\}_{k=1}^{\infty}$ be the Dirichlet eigenvalues and let the associated orthonormal basis of eigenfunctions be $\left\{\phi_{k}\right\}_{k=1}^{\infty}$. Setting the weight function $\phi=-2 \log \phi_{1}$, the variational principle for $(M, g, \phi)$ is that for all $k \geq 1$,

$$
\lambda_{k}-\lambda_{1}=\inf _{\varphi \in \mathcal{C}^{1}(M)}\left\{\frac{\int_{M}|\nabla \varphi|^{2} \phi_{1}^{2}}{\int_{M} \varphi^{2} \phi_{1}^{2}} \mid \varphi \not \equiv 0=\int_{M} \varphi \phi_{j} \phi_{1}, 0 \leq j<k\right\} .
$$

When $k=2$, and the domain $M \subset \mathbb{R}^{n}$, the following variational principle is Corollary 1.3 of [13] and is based on results of [7]. The following proposition is a useful tool.

Proposition 2. For $k \geq 1$, let $\xi_{0}, \ldots, \xi_{k-1}$ be a nontrivial orthogonal set with respect to the weighted $\mathcal{L}^{2}$ measure; that is

$$
\int_{M} \xi_{i} \xi_{j} \mathrm{e}^{-\phi}=0
$$

for $i \neq j$ and $\xi_{i} \not \equiv 0$. Then, we have for the Neumann eigenvalues

$$
\sum_{j=0}^{k} \mu_{j} \leq \sum_{j=0}^{k} \frac{\int_{M}\left|\nabla \xi_{j}\right|^{2} \mathrm{e}^{-\phi}}{\int_{M}\left|\xi_{j}\right|^{2} \phi_{1}^{2}}
$$

The proof is well known and is omitted.

We demonstrate that the difference between the $k$ th and the first Dirichlet eigenvalues is the Neumann eigenvalue of a certain drift Laplacian. This result was known to Singer-Wong-Yau-Yau [22].

Proposition 3. For a bounded domain $M \subset \mathbb{R}^{n}$, let $\left\{\lambda_{k}\right\}_{k=1}^{\infty}$ be the Dirichlet eigenvalues of the Euclidean Laplacian with orthonormal basis of eigenfunctions $\left\{\phi_{k}\right\}_{k=1}^{\infty}$, and let $\left\{\mu_{k}\right\}_{k=0}^{\infty}$ be the Neumann eigenvalues of the drift Laplacian on $M$ with respect to the weight function $-2 \log \phi_{1}$. Then, $\lambda_{k}-\lambda_{1}=\mu_{k-1}$ for all $k \in \mathbb{N}$.

Proof. This follows from the following formula (cf. [19, 20, 22, 29]):

$$
\Delta\left(\frac{\phi_{k}}{\phi_{1}}\right)+2 \nabla \log \phi_{1} \nabla\left(\frac{\phi_{k}}{\phi_{1}}\right)=-\left(\lambda_{k}-\lambda_{1}\right)\left(\frac{\phi_{k}}{\phi_{1}}\right) .
$$


Finally, throughout this paper we will use the following notations: for a function $f(t)$ and fixed $k \geq 0$,

$f(t)=O\left(t^{k}\right)$ as $t \rightarrow 0$ if there exists $C, \delta>0$ such that $|f(t)| \leq C t^{k}$ for all $|t| \leq \delta$;

$$
f(t)=o\left(t^{k}\right) \text { as } t \rightarrow 0 \text { if } \lim _{t \rightarrow 0} \frac{f(t)}{t^{k}}=0 .
$$

Also, throughout this paper, a constant $C$ is independent of $\varepsilon$, but may differ from line to line.

\section{Eigenvalue convergence: a coarse estimate}

In this section, we prove a coarse version of Theorem 1 . Let $(M, g, \phi)$ be the compact Bakry-Émery manifold, with or without boundary, and let

$$
M_{\varepsilon}=\{(x, y) \mid x \in M, \quad 0 \leq y \leq \varepsilon f(x)\} \subset M \times \mathbb{R}^{+}, \quad f(x):=\mathrm{e}^{-\phi(x)} .
$$

Let $\left\{\mu_{k}\right\}_{k=0}^{\infty}$ and $\left\{\psi_{k}\right\}_{k=0}^{\infty}$ be respectively the eigenvalues and eigenfunctions for the drift Laplacian $\Delta$ on $M$ (if $\partial M \neq \emptyset$, we endow it the Neumann boundary condition), and let $\left\{\mu_{k}(\varepsilon)\right\}_{k=0}^{\infty}$ be the eigenvalues for $\tilde{\Delta}=\Delta+\partial_{y}^{2}$ on $M_{\varepsilon}$ with corresponding orthogonal eigenfunctions $\left\{\varphi_{j, \varepsilon}\right\}_{j=0}^{\infty}$. We assume the eigenfunctions are normalized so that

$$
\int_{M_{\varepsilon}} \varphi_{j, \varepsilon} \varphi_{k, \varepsilon}=\varepsilon \delta_{j}^{k} .
$$

In particular, the volume of $M_{\varepsilon}$ is $\varepsilon$. This normalization depends only on $f$ and $M$.

We use $\nabla$ and $\Delta$ as the gradient and Laplace operators, respectively, of $M$, and $\tilde{\nabla}=\left(\nabla, \frac{\partial}{\partial t}\right)$ and $\tilde{\Delta}$ as the gradient and Laplace operators, respectively, of $M_{\varepsilon} \subset$ $M \times \mathbb{R}^{+}$.

We prove the theorem by induction. For $k=0$, the statement of Theorem 1 is trivial. We shall prove the theorem for $k \geq 1$, assuming that for $1, \ldots, k-1$, the theorem has been proven. By a theorem of Uhlenbeck [23], for generic manifold $\mu_{1}, \ldots, \mu_{k}$ are simple; that is, all eigenspaces with respect to the eigenvalues $\mu_{1}, \ldots, \mu_{k}$ are of multiplicity one. Since the eigenvalues are continuous with respect to continuous deformations of the domain, it is sufficient to prove the theorem under this additional assumption.

Lemma 1. Using the above notation, $\mu_{k}(\varepsilon) \leq \mu_{k}+O\left(\varepsilon^{2}\right)$.

Proof. Considering $\psi_{k}$ as functions on $M_{\varepsilon}$, they are orthogonal with respect to the measure $d V_{g} d y$. By Proposition 2, we have

$$
\sum_{j=0}^{k} \mu_{j}(\varepsilon) \leq \sum_{j=0}^{k} \mu_{j} .
$$

By the inductive assumption, we have

$$
\mu_{j} \leq \mu_{j}(\varepsilon)+O\left(\varepsilon^{2}\right)
$$

for all $j<k$. The lemma follows from the above two inequalities. 
For any $0 \leq r \leq \varepsilon$, and for $0 \leq i \leq k$, let

$$
b_{i}(x, r):=\varphi_{i, \varepsilon}(x, r f(x)) \text { and } A_{k}=\sum_{j=0}^{k} \int_{M_{\varepsilon}}\left(\frac{\partial \varphi_{j, \varepsilon}}{\partial y}\right)^{2}(x, y) .
$$

Intuitively, since the domain $M_{\varepsilon}$ is very thin, the eigenfunctions $\varphi_{i, \varepsilon}$ should be almost constant along the $y$-direction. The following lemma confirms this intuition by quantifying the heuristic argument.

Lemma 2. Using the above notations, we have

$$
\left|\int_{M} b_{i}(x, r) b_{j}(x, r) f(x)-\delta_{i}^{j}\right| \leq C \sqrt{\varepsilon A_{k}} \quad \forall 0 \leq i, j \leq k
$$

for all $0 \leq r \leq \varepsilon$.

Proof. ${ }^{4}$ For any $0 \leq r \leq \varepsilon, 0 \leq y \leq \varepsilon f(x)$, and $0 \leq i, j \leq k$,

$$
\begin{aligned}
& \left|b_{i}(x, r) b_{j}(x, r)-\varphi_{i}(x, y) \varphi_{j}(x, y)\right| \leq \int_{0}^{\varepsilon f(x)}\left|\partial_{y}\left(\varphi_{i}(x, y) \varphi_{j}(x, y)\right)\right| d y \\
& \leq \int_{0}^{\varepsilon f(x)}\left(\left|\frac{\partial \varphi_{i}}{\partial y}\right| \cdot\left|\varphi_{j}\right|+\left|\frac{\partial \varphi_{j}}{\partial y}\right| \cdot\left|\varphi_{i}\right|\right)(x, y) d y
\end{aligned}
$$

Note that for any $0 \leq r \leq \varepsilon$,

$$
\varepsilon \int_{M} b_{i}(x, r) b_{j}(x, r) f(x)=\int_{0}^{\varepsilon f(x)} \int_{M} b_{i}(x, r) b_{j}(x, r)=\int_{M_{\varepsilon}} b_{i}(x, r) b_{j}(x, r),
$$

and

$$
\int_{M_{\varepsilon}} \varphi_{i}(x, y) \varphi_{j}(x, y)=\varepsilon \delta_{i}^{j}
$$

Then

$$
\left|\varepsilon \int_{M} b_{i}(x, r) b_{j}(x, r) f(x)-\varepsilon \delta_{i}^{j}\right|=\left|\int_{M_{\varepsilon}}\left(b_{i}(x, r) b_{j}(x, r)-\varphi_{i}(x, y) \varphi_{j}(x, y)\right)\right|,
$$

which by (3.3),

$$
\begin{aligned}
& \leq \int_{M_{\varepsilon}} \int_{0}^{\varepsilon f(x)}\left(\left|\frac{\partial \varphi_{i}}{\partial y}\right| \cdot\left|\varphi_{j}\right|+\left|\frac{\partial \varphi_{j}}{\partial y}\right| \cdot\left|\varphi_{i}\right|\right)(x, t) \\
& \leq \varepsilon\|f\|_{\infty} \int_{M_{\varepsilon}}\left(\left|\frac{\partial \varphi_{i}}{\partial y}\right| \cdot\left|\varphi_{j}\right|+\left|\frac{\partial \varphi_{j}}{\partial y}\right| \cdot\left|\varphi_{i}\right|\right) \\
& \leq \varepsilon\|f\|_{\infty}\left(\sqrt{A_{k}} \cdot\left\|\varphi_{j}\right\|_{L^{2}\left(M_{\varepsilon}\right)}+\sqrt{A_{k}} \cdot\left\|\varphi_{i}\right\|_{L^{2}\left(M_{\varepsilon}\right)}\right) .
\end{aligned}
$$

Since $\left\|\varphi_{i}\right\|_{L^{2}\left(M_{\varepsilon}\right)}=\sqrt{\varepsilon}$,

$$
\left|\varepsilon \int_{M} b_{i}(x, r) b_{j}(x, r) f(x)-\varepsilon \delta_{i}^{j}\right| \leq C \varepsilon^{3 / 2} \sqrt{A_{k}}
$$

\footnotetext{
${ }^{4}$ For simplicity of notation, we drop the subscript $\varepsilon$ from $\varphi$.
} 
Corollary 2. Using the same notations as above, we have

$$
\left|\int_{M} b_{i}(x, r) b_{j}(x, r) f(x)-\delta_{i}^{j}\right| \leq C \varepsilon \quad \forall 0 \leq i, \quad j \leq k, \quad 0 \leq r \leq \varepsilon .
$$

Proof. This follows from the fact that $A_{k} \leq \sum_{j=1}^{k}\left\|\tilde{\nabla} \varphi_{j}\right\|_{L^{2}\left(M_{\varepsilon}\right)}^{2}=\sum_{j=1}^{k} \mu_{j}(\varepsilon) \varepsilon \leq C \varepsilon$, where the constant $C$ depends only on $k$ (not on $\varepsilon$ ).

The following result is a coarse version of Theorem 1.

Lemma 3. Under the same condition as in Theorem 1, and assuming that Theorem 1 is true for $j<k$, we have

$$
\mu_{k} \leq \mu_{k}(\varepsilon)+C\left(\varepsilon^{2}+\sqrt{\varepsilon A_{k}}\right) .
$$

In particular, this estimate and Lemma 1 imply that $\mu_{k}(\varepsilon)=\mu_{k}+O(\varepsilon)$ for all $k \geq 0$.

Proof. Define inductively that $\tilde{b}_{0}(x, r)=b_{0}(x, r)$,

$$
\tilde{b}_{k}(x, r)=b_{k}(x, r)+\sum_{j=0}^{k-1} c_{k j}(r) b_{j}(x, r)
$$

where for any $k \geq 0, c_{k j}(r)$ are functions of $r$ such that

$$
\tilde{b}_{k} \perp b_{1}, \ldots, b_{k-1}
$$

with respect to the measure $f d V_{g}$. The intuition behind defining the functions $\tilde{b}_{k}$ is that the functions $b_{k}$ are almost orthogonal, as proven in Lemma 2, but to obtain the estimate $\mu_{j}(\varepsilon)+O\left(\varepsilon^{2}\right) \leq \mu_{j}$ we need orthogonal test functions to apply Proposition 2 . In the arguments below, we first apply Proposition 2 and then show that the modified functions $\tilde{b}_{k}$ are very close to the original functions $b_{k}$, thereby obtaining the required estimate.

By Proposition 2, we have

$$
\sum_{j=0}^{k} \mu_{j} \leq \sum_{j=0}^{k} \frac{\int_{M}\left|\nabla \tilde{b}_{j}\right|^{2} f}{\int_{M} \tilde{b}_{j}^{2} f}
$$

By Lemma $2, c_{k j}(r) \sim O\left(\sqrt{\varepsilon A_{k}}\right)$ uniformly for $0<r \leq \varepsilon$. Thus by the definition of $\tilde{b}_{k}$, using Lemma 2 again, we have

$$
\sum_{j=0}^{k} \mu_{j} \leq \sum_{j=0}^{k} \frac{\int_{M}\left|\nabla \tilde{b}_{j}\right|^{2} f}{\int_{M} \tilde{b}_{j}^{2} f} \leq\left(1+C \sqrt{\varepsilon A_{k}}\right) \sum_{j=0}^{k} \int_{M}\left|\nabla b_{j}\right|^{2} f .
$$

Since the above inequality holds for all $r$, integrating from 0 to $\varepsilon$, we have

$$
\varepsilon \sum_{j=0}^{k} \mu_{j} \leq\left(1+C \sqrt{\varepsilon A_{k}}\right) \sum_{j=0}^{k} \int_{0}^{\varepsilon} \int_{M}\left|\nabla b_{j}\right|^{2} f .
$$

We compute

$$
\nabla b_{j}(x, r)=\left(\nabla \varphi_{j}\right)(x, r f(x))+r \frac{\partial \varphi_{j}}{\partial y}(x, r f(x)) \nabla f(x)
$$


Using the Cauchy inequality, we get

$$
\left|\nabla b_{j}(x, r)\right|^{2} \leq\left(1+\varepsilon^{2}|\nabla f|^{2}\right)\left|\tilde{\nabla} \varphi_{j, \varepsilon}\right|^{2} .
$$

Therefore,

$$
\int_{0}^{\varepsilon} \int_{M}\left|\nabla b_{j}(x, r)\right|^{2} f(x) \leq\left(1+C \varepsilon^{2}\right) \int_{M_{\varepsilon}}\left|\tilde{\nabla} \varphi_{j, \varepsilon}\right|^{2}=\left(1+C \varepsilon^{2}\right) \mu_{j}(\varepsilon) \varepsilon
$$

for $j \leq k$.

The above estimate together with (3.7) show that

$$
\varepsilon \sum_{j=0}^{k} \mu_{j} \leq \varepsilon\left(1+C \sqrt{\varepsilon A_{k}}\right)\left(1+C \varepsilon^{2}\right) \sum_{j=0}^{k} \mu_{j}(\varepsilon) .
$$

Dividing by $\varepsilon$ and letting $\varepsilon \rightarrow 0$, this estimate together with an induction argument completes the proof of the lemma. The precise estimate $\mu_{k}(\varepsilon)=\mu_{k}+O\left(\varepsilon^{2}\right)$ to complete the proof of Theorem 1 will be demonstrated in the final section.

Proof of Corollary 1. The corollary follows immediately from Lemma 3 and Proposition 3.

\section{A maximum principle}

The Neumann eigenvalues are continuous functions with respect to the manifold $M$. Therefore, to estimate the eigenvalues, we may use an exhaustion of $M$,

$$
M^{\delta}=\{x \in M \mid \operatorname{dist}(x, \partial M) \geq \delta\}, \quad \delta>0 .
$$

On $M^{\delta}, f$ has a positive lower bound. Thus using the variational principle, we may, without loss of generality assume that $f$ is not only positive but is a constant in a neighborhood of $\partial M^{\delta}$. For the rest of the paper, we make such an assumption.

The usual maximum principle for the gradient estimate is the following. Let

$$
H=\frac{1}{2}|\nabla \varphi|^{2}+F(\varphi)
$$

where $F$ is a smooth function of one variable, and let $x_{0}$ be an interior point of $M$ at which $H$ reaches its maximum. Then

$$
0 \geq\left|\nabla^{2} \varphi\right|^{2}+\nabla \varphi \nabla(\Delta \varphi)+\operatorname{Ric}(\nabla \varphi, \nabla \varphi)+F^{\prime}(\varphi) \Delta \varphi+F^{\prime \prime}(\varphi)|\nabla \varphi|^{2} .
$$

The above inequality is very useful for obtaining lower bounds on the first eigenvalue of a Laplace or Schrödinger operator; for more details, we refer to the book [21].

However, it is not appropriate to apply the above maximum principle directly to the manifold $M_{\varepsilon}$ for the following reasons:

(1) $M_{\varepsilon}$ need not be convex, even if $M$ is. As we know, if $M$ is convex, the maximum of $H$ must be reached in the interior of $M$. In general, we do not have such a property for $M_{\varepsilon}$.

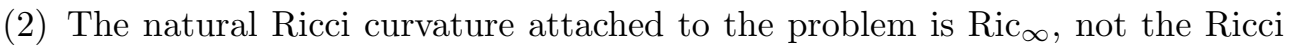
curvature of $M_{\varepsilon}$, which is essentially Ric. 
Remark 2. The choice of $F$ is highly technical. In the Li-Yau's case [15], which is the simplest case, $F(x)=\frac{1}{2} x^{2}$. In Zhong-Yang's case [28], $F$ is (up to a constant)

$$
F(x)=1-x^{2}+a\left(\frac{4}{\pi}\left(\arcsin x+x \sqrt{1-x^{2}}-2 x\right)\right),
$$

where $a$ is a positive constant. More sophisticated choices of $F$ can be found in [16] and $[17]$.

As in the previous sections, we assume $M$ is a compact manifold with or without boundary. Let $U$ be an open set of $M$ and let $\left(x_{1}, \ldots, x_{n}\right)$ be a local coordinates system on $U$. Let $\varphi_{k, \varepsilon}$ be the Neumann eigenfunctions of the eigenvalues $\mu_{k}(\varepsilon)$ with the $L^{2}$ norm normalized to be $\sqrt{\varepsilon}$. We let

$$
\psi(x)=\varphi_{k, \varepsilon}(x, 0), \quad x \in M .
$$

The technical heart of this paper is Theorem 5, which implies the following key results of this section.

Lemma 4. With the above notation, as $\varepsilon \rightarrow 0$,

$$
\begin{aligned}
& \frac{\partial^{2} \varphi_{k, \varepsilon}}{\partial y^{2}}-\nabla \log f(x) \nabla \psi=o(1) \\
& \left(\nabla \psi, \nabla \frac{\partial^{2} \varphi_{k, \varepsilon}}{\partial y^{2}}\right)-\left(\nabla^{2} \log f\right)(\nabla \psi, \nabla \psi)-\nabla^{2} \psi(\nabla \psi, \nabla \log f)=o(1) .
\end{aligned}
$$

Proof. Since $\varphi_{k, \varepsilon}$ satisfies the Neumann condition, we have

$$
\begin{aligned}
& \frac{\partial \varphi_{k, \varepsilon}}{\partial y}(x, 0)=0 \\
& \frac{\partial \varphi_{k, \varepsilon}}{\partial y}(x, \varepsilon f(x))-\varepsilon \nabla f(x) \nabla \varphi_{k, \varepsilon}(x, \varepsilon f(x))=0,
\end{aligned}
$$

for any $x \in M$. Applying the mean value theorem to the above equations, we have

$$
\varepsilon f(x)\left(\frac{\partial^{2} \varphi_{k, \varepsilon}}{\partial y^{2}}(x, \xi(x))-\nabla \log f(x) \nabla \varphi_{k, \varepsilon}(x, \varepsilon f(x))\right)=0,
$$

where $\xi(x) \in(0, \varepsilon f(x))$. Theorem 5 then implies (4.1).

Assume now that at $x$, the local coordinate system is normal. For the second statement, taking partial derivatives with respect to $x_{j}$ in the second equation of (4.3) gives

$$
\begin{aligned}
& \frac{\partial^{2} \varphi_{k, \varepsilon}}{\partial x_{j} \partial y}(x, \varepsilon f(x))+\varepsilon \frac{\partial^{2} \varphi_{k, \varepsilon}}{\partial y^{2}}(x, \varepsilon f(x)) \frac{\partial f}{\partial x_{j}} \\
& -\varepsilon \frac{\partial^{2} f(x)}{\partial x_{i} \partial x_{j}} \frac{\partial \varphi_{k, \varepsilon}}{\partial x_{i}}(x, \varepsilon f(x))-\varepsilon \frac{\partial f(x)}{\partial x_{i}} \frac{\partial^{2} \varphi_{k, \varepsilon}}{\partial x_{i} \partial x_{j}}(x, \varepsilon f(x)) \\
& -\varepsilon^{2} \frac{\partial f}{\partial x_{i}} \frac{\partial^{2} \varphi_{k, \varepsilon}}{\partial x_{i} \partial y}(x, \varepsilon f(x)) \frac{\partial f}{\partial x_{j}}=0 .
\end{aligned}
$$

Since $\partial \varphi_{k, \varepsilon} / \partial y=0$ on $\{y=0\}$, we have

$$
\frac{\partial^{2} \varphi_{k, \varepsilon}}{\partial x_{j} \partial y}(x, 0)=0, \quad \frac{\partial^{3} \varphi_{k, \varepsilon}}{\partial x_{j} \partial x_{i} \partial y}(x, 0)=0 .
$$


The mean value theorem implies

$$
\frac{\partial^{2} \varphi_{k, \varepsilon}}{\partial x_{j} \partial y}(x, \varepsilon f(x))=\varepsilon f(x) \frac{\partial^{3} \varphi_{k, \varepsilon}}{\partial x_{j} \partial y^{2}}(x, \xi(x))
$$

for some $\xi(x) \in(0, \varepsilon f(x))$. Using Theorem 5 again, we have

$$
\begin{gathered}
\varepsilon f(x) \frac{\partial^{3} \varphi_{k, \varepsilon}}{\partial x_{j} \partial y^{2}}(x, 0)+\varepsilon \frac{\partial^{2} \varphi_{k, \varepsilon}}{\partial y^{2}}(x, 0) \frac{\partial f}{\partial x_{j}}-\varepsilon \frac{\partial^{2} f(x)}{\partial x_{i} \partial x_{j}} \frac{\partial \varphi_{k, \varepsilon}}{\partial x_{i}}(x, 0) \\
-\varepsilon \frac{\partial f(x)}{\partial x_{i}} \frac{\partial^{2} \varphi_{k, \varepsilon}}{\partial x_{i} \partial x_{j}}(x, 0)-\varepsilon^{2} \frac{\partial f}{\partial x_{i}} \frac{\partial^{2} \varphi_{k, \varepsilon}}{\partial x_{i} \partial y}(x, 0) \frac{\partial f}{\partial x_{j}}=o(\varepsilon f(x))
\end{gathered}
$$

as $\varepsilon \rightarrow 0$. Thus, we have

$$
\begin{aligned}
& \frac{\partial^{3} \varphi_{k, \varepsilon}}{\partial x_{j} \partial y^{2}}(x, 0)+\frac{\partial^{2} \varphi_{k, \varepsilon}}{\partial y^{2}}(x, 0) \frac{\partial \log f}{\partial x_{j}}-\frac{1}{f} \frac{\partial^{2} f(x)}{\partial x_{i} \partial x_{j}} \frac{\partial \varphi_{k, \varepsilon}}{\partial x_{i}}(x, 0) \\
& -\frac{\partial \log f(x)}{\partial x_{i}} \frac{\partial^{2} \varphi_{k, \varepsilon}}{\partial x_{i} \partial x_{j}}(x, 0)=o(1) .
\end{aligned}
$$

Using (4.1), we get

$$
\begin{aligned}
& \frac{\partial^{3} \varphi_{k, \varepsilon}}{\partial x_{j} \partial y^{2}}(x, 0)+\nabla \log f(x) \nabla \psi(x) \frac{\partial \log f(x)}{\partial x_{j}}-\frac{1}{f(x)} \frac{\partial^{2} f(x)}{\partial x_{i} \partial x_{j}} \frac{\partial \psi(x)}{\partial x_{i}} \\
& -\frac{\partial \log f(x)}{\partial x_{i}} \frac{\partial^{2} \psi}{\partial x_{i} \partial x_{j}}(x)=o(1),
\end{aligned}
$$

which implies (4.2).

For our new maximum principle, we consider

$$
H(x, y)=\frac{1}{2}\left|\tilde{\nabla} \varphi_{k, \varepsilon}\right|^{2}+F\left(\varphi_{k, \varepsilon}\right),
$$

where $F$ is a smooth function of one variable. Assume that $\left(x_{0}, 0\right)$ is the point at which $H$ reaches the maximum on $\{y=0\}$, where $x_{0}$ is in the interior of $M$.

At $\left(x_{0}, 0\right)$, we have

$$
\nabla H\left(x_{0}, 0\right)=0 \text { and } \Delta H\left(x_{0}, 0\right) \leq 0 .
$$

The difficulty is that $H$ satisfies an elliptic equation with respect to $\tilde{\Delta}$, rather than $\Delta$. To obtain the new maximum principle, we need to estimate the second derivative of $H$ in the $y$-direction.

Lemma 5. At $\left(x_{0}, 0\right)$,

$$
\frac{\partial^{2} H}{\partial y^{2}}=\nabla^{2} \log f(\nabla \psi, \nabla \psi)+\left(\frac{\partial^{2} \varphi_{k, \varepsilon}}{\partial y^{2}}\right)^{2}+o(1), \quad \text { as } \quad \varepsilon \rightarrow 0 .
$$

Proof. Using the normal coordinates at $x_{0}$, we have

$$
\frac{\partial H}{\partial y}=\sum_{i=1}^{n} \frac{\partial \varphi_{k, \varepsilon}}{\partial x_{i}} \frac{\partial^{2} \varphi_{k, \varepsilon}}{\partial x_{i} \partial y}+\sum_{i=1}^{n} \frac{\partial \varphi_{k, \varepsilon}}{\partial y} \frac{\partial^{2} \varphi_{k, \varepsilon}}{\partial y^{2}}+F^{\prime}\left(\varphi_{k, \varepsilon}\right) \frac{\partial \varphi_{k, \varepsilon}}{\partial y}
$$


and

$$
\begin{aligned}
\frac{\partial^{2} H}{\partial y^{2}}= & \sum_{i=1}^{n}\left(\frac{\partial^{2} \varphi_{k, \varepsilon}}{\partial x_{i} \partial y}\right)^{2}+\sum_{i=1}^{n} \frac{\partial \varphi_{k, \varepsilon}}{\partial x_{i}} \frac{\partial^{3} \varphi_{k, \varepsilon}}{\partial x_{i} \partial y^{2}} \\
& +\left(\frac{\partial^{2} \varphi_{k, \varepsilon}}{\partial y^{2}}\right)^{2}+\frac{\partial \varphi_{k, \varepsilon}}{\partial y} \frac{\partial^{3} \varphi_{k, \varepsilon}}{\partial y^{3}}+F^{\prime \prime}\left(\varphi_{k, \varepsilon}\right)\left(\frac{\partial \varphi_{k, \varepsilon}}{\partial y}\right)^{2}+F^{\prime}\left(\varphi_{k, \varepsilon}\right) \frac{\partial^{2} \varphi_{k, \varepsilon}}{\partial y^{2}}
\end{aligned}
$$

Since $\varphi_{k, \varepsilon}$ satisfies the Neumann boundary condition, $\frac{\partial \varphi_{k, \varepsilon}}{\partial y}$ and $\frac{\partial^{2} \varphi_{k, \varepsilon}}{\partial x_{i} \partial y}$ vanish on $\{y=0\}$. Thus we have

$$
\frac{\partial^{2} H}{\partial y^{2}}=\sum_{i=1}^{n} \frac{\partial \varphi_{k, \varepsilon}}{\partial x_{i}} \frac{\partial^{3} \varphi_{k, \varepsilon}}{\partial x_{i} \partial y^{2}}+\left(\frac{\partial^{2} \varphi_{k, \varepsilon}}{\partial y^{2}}\right)^{2}+F^{\prime}\left(\varphi_{k, \varepsilon}\right) \frac{\partial^{2} \varphi_{k, \varepsilon}}{\partial y^{2}}
$$

Using Lemma 4, we have

$$
\frac{\partial^{2} H}{\partial y^{2}}=\nabla^{2} \log f(\nabla \psi, \nabla \psi)+\nabla^{2} \psi(\nabla \psi, \nabla \log f)+\left(\frac{\partial^{2} \varphi_{k, \varepsilon}}{\partial y^{2}}\right)^{2}+F^{\prime}(\psi) \frac{\partial^{2} \varphi_{k, \varepsilon}}{\partial y^{2}}+o(1) .
$$

Since at $x_{0}, \nabla H=0$, we have

$$
\sum_{j=1}^{n} \frac{\partial^{2} \psi}{\partial x_{i} \partial x_{j}} \frac{\partial \psi}{\partial x_{j}}+F^{\prime}(\psi) \frac{\partial \psi}{\partial x_{i}}=0, \quad \text { for each } \quad 1 \leq i \leq n
$$

Using the above equality and Lemma 4, the second and fourth terms on the right side of the expression for $\frac{\partial^{2} H}{\partial y^{2}}$ cancel.

Theorem 2 (Maximum Principle). With the above notations, we have at $\left(x_{0}, 0\right)$

$$
o(1) \geq\left|\nabla^{2} \psi\right|^{2}+\nabla \psi \nabla\left(\tilde{\Delta} \varphi_{k, \varepsilon}\right)+\operatorname{Ric}_{\infty}(\nabla \psi, \nabla \psi)+F^{\prime}(\psi) \tilde{\Delta} \varphi_{k, \varepsilon}+F^{\prime \prime}(\psi)|\nabla \psi|^{2} .
$$

Proof. By the Bochner formula, we have

$$
\begin{aligned}
\tilde{\Delta} H= & \left|\tilde{\nabla}^{2} \varphi_{k, \varepsilon}\right|^{2}+\operatorname{Ric}\left(\tilde{\nabla} \varphi_{k, \varepsilon}, \tilde{\nabla} \varphi_{k, \varepsilon}\right)+\tilde{\nabla} \varphi_{k, \varepsilon} \tilde{\nabla}\left(\tilde{\Delta} \varphi_{k, \varepsilon}\right) \\
& +F^{\prime}\left(\varphi_{k, \varepsilon}\right) \tilde{\Delta} \varphi_{k, \varepsilon}+F^{\prime \prime}\left(\varphi_{k, \varepsilon}\right)\left|\tilde{\nabla} \varphi_{k, \varepsilon}\right|^{2} .
\end{aligned}
$$

On $\{y=0\}$, we have

$$
\begin{aligned}
\left|\tilde{\nabla}^{2} \varphi_{k, \varepsilon}\right|^{2} & =\left|\nabla^{2} \psi\right|^{2}+\left(\frac{\partial^{2} \varphi_{k, \varepsilon}}{\partial y^{2}}\right)^{2}, \\
\operatorname{Ric}\left(\tilde{\nabla} \varphi_{k, \varepsilon}, \tilde{\nabla} \varphi_{k, \varepsilon}\right) & =\operatorname{Ric}(\nabla \psi, \nabla \psi), \\
\tilde{\Delta} \varphi_{k, \varepsilon} & =\Delta \psi+\frac{\partial^{2} \varphi_{k, \varepsilon}}{\partial y^{2}},
\end{aligned}
$$

Thus, we have

$$
\begin{aligned}
\tilde{\Delta} H= & \left|\nabla^{2} \psi\right|^{2}+\left(\frac{\partial^{2} \varphi_{k, \varepsilon}}{\partial y^{2}}\right)^{2}+\operatorname{Ric}(\nabla \psi, \nabla \psi) \\
& +\nabla \psi \nabla\left(\tilde{\Delta} \varphi_{k, \varepsilon}\right)+F^{\prime}(\psi) \tilde{\Delta} \varphi_{k, \varepsilon}+F^{\prime \prime}(\psi)|\nabla \psi|^{2}
\end{aligned}
$$


Using Lemma 5, noting that at $\left(x_{0}, 0\right)$

$$
\tilde{\Delta} H=\Delta H+\frac{\partial^{2} H}{\partial y^{2}} \leq \frac{\partial^{2} H}{\partial y^{2}}
$$

completes the proof.

4.1. Applications. Our work not only has applications to Bakry-Émery geometry but also to Ricci solitons. We recall the main result of Futaki and Sano [9].

Theorem 3 (Futaki-Sano). Let $M^{n}$ be a compact smooth manifold of dimension at least 4. If $g$ is a non-trivial gradient shrinking Ricci soliton on $M$ (see definition 1.1 of [9]), then the diameter of $M$ with respect to $g$ is bounded below by $\frac{10 \pi}{13 \sqrt{\gamma}}$, where $\gamma$ is a constant determined by $g$.

This result is proven by using Ling's gradient estimates [16] to demonstrate a lower bound for the first non-zero eigenvalue of a certain Bakry-Émery Laplacian. Our Principle shows that one may directly apply Ling's estimates to the Bakry-Émery Laplacian to obtain the result. It is reasonable to expect that one may similarly express elliptic geometric equations, like the Ricci soliton equation, in terms of a Bakry-Émery Laplacian and exploit the eigenvalue estimates from Riemannian geometry together with our Principle to produce interesting results.

Another application arises from the so-called fundamental gap: the difference between the first two Dirichlet eigenvalues of a domain in $\mathbb{R}^{n}$. Andrews and Clutterbuck [2] recently demonstrated an optimal lower bound of $3 \pi^{2} / d^{2}$ for the fundamental gap of any convex domain in $\mathbb{R}^{n}$ with diameter $d$. By Proposition 3 , the fundamental gap can be interpreted as the first Neumann eigenvalue on certain Bakry-Émery manifold, and in particular, techniques of $[1,2]$ together with our work imply the following.

Theorem 4. Let $\Omega \subset \mathbb{R}^{n}$ be a convex domain with piecewise smooth boundary and diameter $d$. Let $f \in \mathcal{C}^{2}(\bar{\Omega})$. If $f$ satisfies

$$
(\nabla \log f(y)-\nabla \log f(x)) \cdot \frac{y-x}{|y-x|} \geq \frac{4 \pi}{d} \tan \left(\frac{\pi|y-x|}{d}\right) \quad \forall \quad x \neq y \text { in } \Omega,
$$

then the first non-trivial Neumann eigenvalue of the Bakry-Émery Laplacian with respect to the weight function $\phi=-\log \left(f^{2}\right)$ is bounded below by $3 \pi^{2} / d^{2}$. Moreover, the first Neumann eigenfunction for the Euclidean Laplacian on

$$
\Omega_{\varepsilon}:=\left\{(x, y) \mid x \in \Omega, 0 \leq y \leq \varepsilon f^{2}(x)\right\} \subset \mathbb{R}^{n+1}
$$

is bounded below by $3 \pi^{2} / d^{2}-C \varepsilon^{2}$, where $C$ is a fixed constant that depends only on $n$ and $\Omega$.

\section{The approximation of eigenfunctions}

It is not hard to write down the eigenfunctions formally. Let $\varphi$ be a Neumann eigenfunction of $M_{\varepsilon}$ with eigenvalue $\lambda$. Write

$$
\varphi=\sum_{k=0}^{\infty} y^{k} \varphi_{k}
$$


where $\varphi_{k}$ are functions on $M$. Then we have (formally)

$$
\Delta \varphi_{k}+\lambda \varphi_{k}+(k+1)(k+2) \varphi_{k+2}=0
$$

for all $k \geq 0$. Since $\partial \varphi / \partial y=0$ on $\{y=0\}$, we have $\varphi_{1}=0$ and hence $\varphi_{2 k+1}=0$ for all $k$. Let

$$
H \varphi=-\Delta \varphi-\lambda \varphi
$$

Then

$$
\varphi_{2 k+2}=\frac{H \varphi_{2 k}}{(2 k+1)(2 k+2)}=\frac{H^{k+1} \varphi_{0}}{(2 k+2) !} .
$$

Formally, we have

$$
\varphi=\sum_{k=0}^{\infty} \frac{y^{2 k} H^{k}}{(2 k) !} \varphi_{0}=\cosh (y \sqrt{H}) \varphi_{0} .
$$

The differential equation for $\varphi_{0}$ follows from the Neumann boundary condition

$$
\sqrt{H} \sinh (\varepsilon f(x) \sqrt{H}) \varphi_{0}-\varepsilon \nabla f \cdot \nabla\left(\left.\cosh (y \sqrt{H}) \varphi_{0}\right|_{y=\varepsilon f(x)}\right)=0 .
$$

We are not able to prove the full regularity of the above equation at this moment. But a partial solution, namely, a good approximation to the eigenfunctions, is enough for our application. Very roughly speaking, in this section, we prove

$$
\varphi=\varphi_{0}+y^{2} \varphi_{2}+O\left(\varepsilon^{3}\right)
$$

To state our results precisely, we recall the global Schauder estimates $[12]^{*}$ Theorem 6.6, Theorem 6.30 and the interpolation inequalities.

We let

$$
\begin{aligned}
B_{\mathrm{I}} & =\left\{(x, y) \in M_{\varepsilon} \mid y=0\right\} ; \\
B_{\mathrm{II}} & =\left\{(x, y) \in M_{\varepsilon} \mid y=\varepsilon f(x)\right\} ; \\
B_{\mathrm{III}} & =\left\{(x, y) \in M_{\varepsilon} \mid x \in \partial M\right\} .
\end{aligned}
$$

Then $B_{\mathrm{I}} \cup B_{\mathrm{II}} \cup B_{\mathrm{III}}=\partial M_{\varepsilon}$.

Let

$$
u_{1}=\left.u\right|_{\partial M_{\varepsilon}} \text { and } u_{2}=\left.\frac{\partial u}{\partial n}\right|_{\partial M_{\varepsilon}} \text { on the smooth part of } \partial M_{\varepsilon} .
$$

Define the weighted Hölder norm by

$$
\|u\|_{C_{\varepsilon}^{k, \alpha}}=\varepsilon^{k+\alpha}[u]_{C^{k, \alpha}}+\cdots+\varepsilon^{\alpha}[u]_{C^{\alpha}}+\|u\|_{C^{0}}
$$

and $\|u\|_{C_{\varepsilon}^{\alpha}}=\|u\|_{C_{\varepsilon}^{0, \alpha}}$, where [ $]_{C^{k, \alpha}}$ are the standard notations defined in [12]. Using these weighted norms, the constants in the Schauder estimates on $M_{\varepsilon}$ are independent of $\varepsilon$. Let $0<\alpha<1$. Let $L$ be a second order uniform elliptic operator with $C^{\alpha}$-bounded coefficients. Then we have the following version of global Schauder estimates on $M_{\varepsilon}$

$$
\|u\|_{C_{\varepsilon}^{2, \alpha}} \leq C\left(\|u\|_{C^{0}}+\varepsilon^{2}\|L u\|_{C_{\varepsilon}^{\alpha}}+\varepsilon\left\|u_{2}\right\|_{C_{\varepsilon}^{1, \alpha}}\right),
$$

and

$$
\|u\|_{C_{\varepsilon}^{2, \alpha}} \leq C\left(\|u\|_{C^{0}}+\varepsilon^{2}\|L u\|_{C_{\varepsilon}^{\alpha}}+\varepsilon\left\|u_{2}\right\|_{C_{\varepsilon}^{1, \alpha}\left(B_{\mathrm{III}}\right)}+\left\|u_{1}\right\|_{C_{\varepsilon}^{2, \alpha}\left(B_{\mathrm{I}} \cup B_{\mathrm{II}}\right)}\right) .
$$


The Sobolev inequality on $M_{\varepsilon}$ is

$$
\left(\int_{M_{\varepsilon}}|u|^{2 \frac{n+1}{n-1}}\right)^{\frac{n-1}{n+1}} \leq C \varepsilon^{-\frac{2}{n+1}}\left(\int_{M_{\varepsilon}}|\nabla u|^{2}+\int_{M_{\varepsilon}}|u|^{2}\right) .
$$

Define the Hölder norm in the $y$-direction to be

$$
[u]_{C_{y}^{\alpha}}=\max _{x \in M} \sup _{0 \leq y_{1}, y_{2} \leq \varepsilon f(x)} \frac{\left|u\left(x, y_{1}\right)-u\left(x, y_{2}\right)\right|}{\left|y_{1}-y_{2}\right|^{\alpha}} .
$$

Then we have the following.

Theorem 5. For $M_{\varepsilon}$ defined by (3.1) such that $f(x)=e^{-\phi(x)}$ is constant in a neighborhood of $\partial M$, the Neumann eigenfunctions $\varphi_{k, \varepsilon}$ of $M_{\varepsilon}$ satisfy

$$
\begin{aligned}
& \nabla \varphi_{k, \varepsilon}=O(1), \\
& \left\|\frac{\partial^{2} \varphi_{k, \varepsilon}}{\partial y^{2}}\right\|_{C_{y}^{\alpha}}+\left\|\frac{\partial^{2} \varphi_{k, \varepsilon}}{\partial x_{j} \partial y}\right\|_{C_{y}^{\alpha}}=O(1), \quad 1 \leq j \leq n \\
& \left\|\frac{\partial^{3} \varphi_{k, \varepsilon}}{\partial x_{j} \partial y^{2}}\right\|_{C_{y}^{\alpha}}+\left\|\frac{\partial^{3} \varphi_{k, \varepsilon}}{\partial x_{i} \partial x_{j} \partial y}\right\|_{C_{y}^{\alpha}}=O(1), \quad 1 \leq i, j \leq n
\end{aligned}
$$

for any $0<\alpha<1$, where $\left(x_{1}, \ldots, x_{n}\right)$ is any local coordinate system of $M$.

As defined in Section 3, let $\left\{\mu_{k}\right\}_{k=0}^{\infty}$ and $\left\{\psi_{k}\right\}_{k=0}^{\infty}$ be respectively the eigenvalues and eigenfunctions for the drift Laplacian. Moreover, we assume that $\left\{\psi_{k}\right\}_{k=0}^{\infty}$ is an orthonormal basis for the space of $L^{2}$ functions of $M$ with respect to the weighted measure $\mathrm{e}^{-\phi} d V_{g}$.

Define the functions

$$
\eta_{k}:=-\nabla \phi \nabla \psi_{k}
$$

on $M$, and let

$$
U_{k}:=\psi_{k}+\frac{1}{2} y^{2} \eta_{k}
$$

be functions on $M_{\varepsilon}$. By our definition of $f(x)=\mathrm{e}^{-\phi(x)}, \eta_{k}$ are smooth up to the boundary. Note that since $\psi_{k}$ is a Bakry-Émery eigenfunction, it satisfies

$$
\Delta \psi_{k}+\eta_{k}=-\mu_{k} \psi_{k}
$$

Since $\psi_{k}$ and $\eta_{k}$ are independent of $y$,

$$
\tilde{\Delta} U_{k}=-\mu_{k} \psi_{k}+\frac{1}{2} y^{2} \Delta \eta_{k}=-\mu_{k} U_{k}+\frac{1}{2} y^{2}\left(\Delta \eta_{k}+\mu_{k} \eta_{k}\right) .
$$

We compute directly,

$$
\left.\frac{\partial U_{k}}{\partial n}\right|_{\partial M_{\varepsilon}}= \begin{cases}0 & \text { on } B_{\mathrm{I}} \cup B_{\mathrm{III}} \\ -\frac{\varepsilon^{3} f^{2} \nabla f \nabla \eta_{k}}{2\left(1+\varepsilon^{2}|\nabla f|^{2}\right)^{1 / 2}} & \text { on } B_{\mathrm{II}} .\end{cases}
$$

Define

$$
w_{k, \varepsilon}=\alpha_{k, k} \varphi_{k, \varepsilon}+U_{k}+\sum_{j=0}^{k-1} \alpha_{k, j} \varphi_{j, \varepsilon}
$$

where $\alpha_{k, j}$ are defined such that $w_{k, \varepsilon} \perp \varphi_{0, \varepsilon}, \ldots, \varphi_{k, \varepsilon}$ in $\mathcal{L}^{2}\left(M_{\varepsilon}\right)$. 
Heuristically, by (5.5) and (5.6), $U_{k}$ should be very close to the eigenfunction $\varphi_{k, \varepsilon}$, and the function $w_{k, \varepsilon}$ should be approximately the difference between $U_{k}$ and $\varphi_{k, \varepsilon}$. In what follows, we prove that $w_{k, \varepsilon}$ and its $y$ derivative (denoted $r_{k, \varepsilon}$ in (5.12) below) are indeed small, thereby rigorously demonstrating our intuitive heuristic.

The following inequality will be used repeatedly in the rest of the paper: let $\varphi$ be a function on $M_{\varepsilon}$. Then for any $p>0$, we have

$$
\int_{B_{I I}}|\varphi|^{p} \leq C\left(\frac{1}{\varepsilon} \int_{M_{\varepsilon}}|\varphi|^{p}+p \sqrt{\int_{M_{\varepsilon}}|\varphi|^{p-1}} \cdot \sqrt{\int_{M_{\varepsilon}}|\varphi|^{p-1}|\nabla \varphi|^{2}}\right) .
$$

To prove (5.7), we observe that for any $0 \leq y \leq \varepsilon f(x)$, we have

$$
|\varphi|^{p}(x, \varepsilon f(x)) \leq|\varphi|^{p}(x, y)+p \int_{0}^{\varepsilon f(x)}|\varphi|^{p-1}|\nabla \varphi| d y .
$$

Integrating over $M_{\varepsilon}$ to both sides of the above equation and using the Cauchy-Schwarz inequality implies (5.7).

Lemma 6. For all $j<k, \alpha_{k, j}=O\left(\varepsilon^{2}\right)$, and $\alpha_{k, k}=O(1)$.

Proof. Integrating by parts, (5.5) and (5.6) give

$$
\begin{aligned}
\mu_{j}(\varepsilon) \int_{M_{\varepsilon}} \varphi_{j, \varepsilon} U_{k} & =-\int_{M_{\varepsilon}} \tilde{\Delta} \varphi_{j, \varepsilon} U_{k}=\int_{\partial M_{\varepsilon}} \varphi_{j, \varepsilon} \frac{\partial U_{k}}{\partial n}-\int_{M_{\varepsilon}} \varphi_{j, \varepsilon} \tilde{\Delta} U_{k} \\
& =\int_{\partial M_{\varepsilon}} \varphi_{j, \varepsilon} \frac{\partial U_{k}}{\partial n}+\mu_{k} \int_{M_{\varepsilon}} \varphi_{j, \varepsilon} U_{k}-\frac{1}{2} \int_{M_{\varepsilon}} \varphi_{j, \varepsilon} y^{2}\left(\Delta \eta_{k}+\mu_{k} \eta_{k}\right) .
\end{aligned}
$$

Thus by (5.6) again, we have

$$
\left(\mu_{j}(\varepsilon)-\mu_{k}\right) \int_{M_{\varepsilon}} \varphi_{j, \varepsilon} U_{k}=-\int_{B_{I I}} \varphi_{j, \varepsilon} \frac{\partial U_{k}}{\partial n}-\frac{1}{2} \int_{M_{\varepsilon}} \varphi_{j, \varepsilon} y^{2}\left(\Delta \eta_{k}+\mu_{k} \eta_{k}\right) .
$$

We clearly have

$$
\left|\int_{M_{\varepsilon}} \varphi_{j, \varepsilon} y^{2}\left(\Delta \eta_{k}+\mu_{k} \eta_{k}\right)\right| \leq C \sqrt{\int_{M_{\varepsilon}} y^{4}} \cdot \sqrt{\int_{M_{\varepsilon}}\left|\varphi_{j, \varepsilon}\right|^{2}}=O\left(\varepsilon^{3}\right) .
$$

Using (5.7) for $p=1$, we have

$$
\left|\int_{B_{I I}} \varphi_{j, \varepsilon} \frac{\partial U_{k}}{\partial n}\right| \leq C \varepsilon^{3}
$$

By the generic assumption of the manifold $M$ and Lemma $3, \mu_{j}(\varepsilon)=\mu_{j}+O(\varepsilon)<\mu_{k}$ for all $j<k$ for $\varepsilon$ sufficiently small. Thus, dividing by $\left(\mu_{j}(\varepsilon)-\mu_{k}\right)$ in (5.8) gives

$$
\int_{M_{\varepsilon}} \varphi_{j, \varepsilon} U_{k}=O\left(\varepsilon^{3}\right) \Longrightarrow \alpha_{k, j}=O\left(\varepsilon^{2}\right)
$$

That $\alpha_{k, k}$ is bounded follows from its definition. 
A straightforward computation gives

$$
\begin{gathered}
F_{1}:=\tilde{\Delta} w_{k, \varepsilon}+\mu_{k}(\varepsilon) w_{k, \varepsilon} \\
=\left(\mu_{k}(\varepsilon)-\mu_{k}\right) U_{k}+\frac{1}{2} y^{2}\left(\Delta \eta_{k}+\mu_{k} \eta_{k}\right)+\sum_{j=0}^{k-1} \alpha_{k, j}\left(\mu_{k}(\varepsilon)-\mu_{j}(\varepsilon)\right) \varphi_{j, \varepsilon}
\end{gathered}
$$

with the boundary conditions

$$
\frac{\partial w_{k, \varepsilon}}{\partial n}= \begin{cases}0 & \text { on } B_{\mathrm{I}} \cup B_{\mathrm{III}}, \\ \frac{\partial U_{k}}{\partial n}=O\left(\varepsilon^{3}\right) & \text { on } B_{\mathrm{II}}\end{cases}
$$

Let

Then

$$
r_{k, \varepsilon}=\frac{\partial w_{k, \varepsilon}}{\partial y}
$$

$$
\begin{gathered}
F_{2}:=\tilde{\Delta} r_{k, \varepsilon}+\mu_{k}(\varepsilon) r_{k, \varepsilon} \\
=\left(\mu_{k}(\varepsilon)-\mu_{k}\right) y \eta_{k}+y\left(\Delta \eta_{k}+\mu_{k} \eta_{k}\right)+\sum_{j=0}^{k-1} \alpha_{k, j}\left(\mu_{k}(\varepsilon)-\mu_{j}(\varepsilon)\right) \frac{\partial \varphi_{j, \varepsilon}}{\partial y}
\end{gathered}
$$

with the boundary conditions

$$
\begin{cases}r_{k, \varepsilon}=0 & \text { on } B_{\mathrm{I}}, \\ r_{k, \varepsilon}=\varepsilon \nabla f(x) \nabla w_{k, \varepsilon}(x, \varepsilon f(x))+\mathcal{O}\left(\varepsilon^{3}\right) & \text { on } B_{\mathrm{II}} \\ \frac{\partial r_{k, \varepsilon}}{\partial n}=0 & \text { on } B_{\mathrm{III}} .\end{cases}
$$

Inductively, we assume that the Theorem 5 is true for $j \leq k-1$. Then by Lemma 3 and Lemma 6, we have

$$
\left\|F_{1}\right\|_{C_{\varepsilon}^{\alpha}}=O\left(B_{k}\right) \text { and }\left\|F_{2}\right\|_{C_{\varepsilon}^{\alpha}}=O(\varepsilon),
$$

where

$$
B_{k}=\varepsilon^{2}+\sqrt{\varepsilon A_{k}} .
$$

Lemma 7. With the above notations, we have

$$
\left\|w_{k, \varepsilon}\right\|_{L^{2}\left(M_{\varepsilon}\right)}=O\left(\varepsilon^{1 / 2} B_{k}\right) \text { and }\left\|\tilde{\nabla} w_{k, \varepsilon}\right\|_{L^{2}\left(M_{\varepsilon}\right)}=O\left(\varepsilon^{1 / 2} B_{k}\right) \text {. }
$$

Proof. Multiplying both sides of (5.11) by $w_{k, \varepsilon}$ and integrating by parts, using (5.15), we get

$$
\begin{aligned}
& \left\|\nabla w_{k, \varepsilon}\right\|_{L^{2}\left(M_{\varepsilon}\right)}^{2}-\mu_{k}(\varepsilon)\left\|w_{k, \varepsilon}\right\|_{L^{2}\left(M_{\varepsilon}\right)}^{2} \\
& \leq C B_{k} \cdot\left\|w_{k, \varepsilon}\right\|_{L^{1}\left(M_{\varepsilon}\right)}+\left|\int_{B_{\mathrm{II}}} w_{k, \varepsilon} \frac{\partial w_{k, \varepsilon}}{\partial n}\right| .
\end{aligned}
$$

By (5.7), we have

$$
\left|\int_{B_{I I}} w_{k, \varepsilon} \frac{\partial w_{k, \varepsilon}}{\partial n}\right|=\left|\int_{B_{I I}} w_{k, \varepsilon} \frac{\partial U_{k}}{\partial n}\right| \leq C \varepsilon^{5 / 2}\left(\left\|w_{k, \varepsilon}\right\|_{L^{2}\left(M_{\varepsilon}\right)}+\left\|\nabla w_{k, \varepsilon}\right\|_{L^{2}\left(M_{\varepsilon}\right)}\right) .
$$

Thus, we have

$$
\|\nabla w\|_{L^{2}\left(M_{\varepsilon}\right)}^{2}-\mu_{k}(\varepsilon)\|w\|_{L^{2}\left(M_{\varepsilon}\right)}^{2} \leq C \varepsilon^{5 / 2}\left(\left\|w_{k, \varepsilon}\right\|_{L^{2}\left(M_{\varepsilon}\right)}+\left\|\nabla w_{k, \varepsilon}\right\|_{L^{2}\left(M_{\varepsilon}\right)}\right) .
$$


By the Poincaré inequality, we have

$$
\mu_{k+1}(\varepsilon) \int_{M_{\varepsilon}}\left|w_{k, \varepsilon}\right|^{2} \leq \int_{M_{\varepsilon}}\left|\nabla w_{k, \varepsilon}\right|^{2}
$$

The lemma is proved since by our "generic" assumption, there is a gap between $\mu_{k+1}(\varepsilon)$ and $\mu_{k}(\varepsilon)$ that is independent of $\varepsilon$.

Using the above lemma we shall complete the proof of Theorem 1.

Proof of Theorem 1. Since $B_{k}=O(\varepsilon)$, by the above lemma, $\left\|w_{k, \varepsilon}\right\|_{L^{2}\left(M_{\varepsilon}\right)}=$ $O\left(\varepsilon^{3 / 2}\right)$. It follows that

$$
A_{k}=\sum_{j=1}^{k} \int_{M_{\varepsilon}}\left|\frac{\partial \varphi_{k, \varepsilon}}{\partial y}\right|^{2}=O\left(\varepsilon^{3}\right)
$$

and thus Theorem 1 follows from Lemma 3.

Corollary 3. With the above notations, we have

$$
\left\|w_{k, \varepsilon}\right\|_{L^{2}\left(M_{\varepsilon}\right)}=O\left(\varepsilon^{5 / 2}\right) \quad \text { and } \quad\left\|\tilde{\nabla} w_{k, \varepsilon}\right\|_{L^{2}\left(M_{\varepsilon}\right)}=O\left(\varepsilon^{5 / 2}\right) .
$$

Now we work towards the proof of Theorem 5 .

Lemma 8. $w_{k, \varepsilon}=O\left(\varepsilon^{2}\right)$

Proof. We prove the lemma using Moser iteration. By (5.15) and Theorem $1, F_{1}=$ $O\left(\varepsilon^{2}\right)$ is a bounded function. Using integration by parts, we get

$p \int_{M_{\varepsilon}}\left|w_{k, \varepsilon}\right|^{p-1}\left|\nabla w_{k, \varepsilon}\right|^{2} \leq \int_{M_{\varepsilon}}\left|F_{1}\right|\left|w_{k, \varepsilon}\right|^{p}+\mu_{k}(\varepsilon) \int_{M_{\varepsilon}}\left|w_{k, \varepsilon}\right|^{p+1}+\int_{B_{\mathrm{II}}}\left|w_{k, \varepsilon}\right|^{p}\left|\frac{\partial w_{k, \varepsilon}}{\partial n}\right|$.

By (5.6), we have

$$
\int_{B_{I I}}\left|w_{k, \varepsilon}\right|^{p}\left|\frac{\partial w_{k, \varepsilon}}{\partial n}\right| \leq C \varepsilon^{3} \int_{B_{\mathrm{II}}}\left|w_{k, \varepsilon}\right|^{p}
$$

By (5.7), we have

$$
\int_{B_{I I}}\left|w_{k, \varepsilon}\right|^{p} \leq\left(\frac{1}{\varepsilon} \int_{M_{\varepsilon}}\left|w_{k, \varepsilon}\right|^{p}+p \sqrt{\int_{M_{\varepsilon}}\left|w_{k, \varepsilon}\right|^{p-1}} \cdot \sqrt{\int_{M_{\varepsilon}}\left|w_{k, \varepsilon}\right|^{p-1}\left|\nabla w_{k, \varepsilon}\right|^{2}}\right) .
$$

It follows that

$$
\int_{B_{I I}}\left|w_{k, \varepsilon}\right|^{p}\left|\frac{\partial w_{k, \varepsilon}}{\partial n}\right| \leq \frac{1}{4} p \int_{M_{\varepsilon}}\left|w_{k, \varepsilon}\right|^{p-1}\left|\nabla w_{k, \varepsilon}\right|^{2}+\varepsilon^{6} p \int_{M_{\varepsilon}}\left|w_{k, \varepsilon}\right|^{p-1}+\varepsilon^{2} \int_{M_{\varepsilon}}\left|w_{k, \varepsilon}\right|^{p} .
$$

By the Young's inequality, we have

$$
\varepsilon^{2}\left|w_{k, \varepsilon}\right|^{p}+\varepsilon^{6}\left|w_{k, \varepsilon}\right|^{p-1} \leq\left|w_{k, \varepsilon}\right|^{p+1}+\varepsilon^{2(p+1)} .
$$

Thus, we have

$$
\int_{B_{\mathrm{II}}}\left|w_{k, \varepsilon}\right|^{p}\left|\frac{\partial w_{k, \varepsilon}}{\partial n}\right| \leq \frac{p}{4} \int_{M_{\varepsilon}}\left|w_{k, \varepsilon}\right|^{p-1}\left|\nabla w_{k, \varepsilon}\right|^{2}+C p\left(\left(\int_{M_{\varepsilon}}\left|w_{k, \varepsilon}\right|^{p+1}\right)+\varepsilon^{2(p+1)+1}\right)
$$


from which we have

$$
p \int_{M_{\varepsilon}}\left|w_{k, \varepsilon}\right|^{p-1}\left|\nabla w_{k, \varepsilon}\right|^{2} \leq C p\left(\left(\int_{M_{\varepsilon}}\left|w_{k, \varepsilon}\right|^{p+1}\right)+\varepsilon^{2(p+1)+1}\right) .
$$

Using the above inequality and the Sobolev inequality (5.3), we have

$$
\left(\int_{M_{\varepsilon}}\left|w_{k, \varepsilon}\right|^{(p+1) \frac{n+1}{n-1}}\right)^{\frac{n-1}{n+1}} \leq C p^{2} \varepsilon^{-\frac{2}{n+1}}\left(\int_{M_{\varepsilon}}\left|w_{k, \varepsilon}\right|^{p+1}+\varepsilon^{2(p+1)+1}\right) .
$$

We thus have

$$
\left(\int_{M_{\varepsilon}}\left|w_{k, \varepsilon}\right|^{(p+1) \frac{n+1}{n-1}}+\varepsilon^{2(p+1) \frac{n+1}{n-1}+1}\right)^{\frac{n-1}{n+1}} \leq C p^{2} \varepsilon^{-\frac{2}{n+1}}\left(\int_{M_{\varepsilon}}\left|w_{k, \varepsilon}\right|^{p+1}+\varepsilon^{2(p+1)+1}\right) .
$$

Let

$$
a_{k}=2\left(\frac{n+1}{n-1}\right)^{k}
$$

for $k \geq 0$. Let

$$
b_{k}=\left(\int_{M_{\varepsilon}}\left|w_{k, \varepsilon}\right|^{a_{k}}+\varepsilon^{2\left(a_{k}\right)+1}\right)^{1 / a_{k}} .
$$

Then we have

$$
b_{k+1} \leq\left(C\left(a_{k}\right)^{2} \varepsilon^{-\frac{2}{n+1}}\right)^{1 / a_{k}} b_{k} .
$$

The standard iteration process shows that

$$
\left\|w_{k, \varepsilon}\right\|_{C^{0}} \leq C \varepsilon^{-\frac{1}{n+1} \sum 1 / a_{k}} b_{0} \leq C \varepsilon^{2} .
$$

Lemma 9. $r_{k, \varepsilon}=O\left(\varepsilon^{2}\right)$.

Proof. We could run Moser iteration again to get the estimate. However, the following proof using the maximum principle seems to be simpler. Using (5.1), (5.15), and the boundary conditions (5.14), we have

$$
\left\|w_{k, \varepsilon}\right\|_{C_{\varepsilon}^{2, \alpha}} \leq \varepsilon^{2}
$$

which implies that

$$
\left|\tilde{\nabla}^{2} w_{k, \varepsilon}\right| \leq C, \quad\left|\tilde{\nabla} w_{k, \varepsilon}\right| \leq C \varepsilon
$$

by the interpolation inequalities. For $C>0$ large, by (5.15), we have

$$
\tilde{\Delta}\left(r_{k, \varepsilon}+C y^{2}\right)>0 \text {. }
$$

Since $r_{k, \varepsilon}=0$ on $B_{\mathrm{I}}, \frac{\partial r_{k, \varepsilon}}{\partial n}=0$ on $B_{\mathrm{III}}$. By the maximum principle, we only need to estimate the maximum value of $r_{k, \varepsilon}$ on $B_{\mathrm{II}}$. But on $B_{I I}, r_{k, \varepsilon}=\varepsilon \nabla f \nabla w_{k, \varepsilon}+O\left(\varepsilon^{3}\right)=$ $O\left(\varepsilon^{2}\right)$. Thus, we have $r_{k, \varepsilon} \leq C \varepsilon^{2}$. Similarly estimating $r_{k, \varepsilon}-C y^{2}$ gives the other side of the inequality.

Lemma 10. $\left.|| r_{k, \varepsilon}\right|_{C_{\varepsilon}^{2, \alpha}}=O\left(\varepsilon^{2}\right)$, and $\left|\tilde{\nabla}^{2} w_{k, \varepsilon}\right|=O(\varepsilon)$. 
Proof. For fixed $y$, let $w=w_{k, \varepsilon}$ and let $h=w(x, y f(x))$. Then $h$ satisfies the equation

$$
\begin{aligned}
& \Delta h+\mu_{k}(\varepsilon) h \\
& =-\left(1-y^{2}|\nabla f|^{2}\right) \frac{\partial r_{k, \varepsilon}}{\partial y}+2 y\left\langle\nabla f, \nabla r_{k, \varepsilon}\right\rangle+y r_{k, \varepsilon} \Delta f+F_{1}(x, y f(x)) .
\end{aligned}
$$

Let $\Omega \subset M$ such that on $M \backslash \Omega, f$ is identically equal to a positive constant $\delta$. By the Schauder interior estimate, we have

$$
\|h\|_{C^{3, \alpha}(\Omega)} \leq C\left(\varepsilon^{2}+\left\|r_{k, \varepsilon}\right\|_{C^{2, \alpha}}\right) .
$$

Note that the above $C^{2, \alpha}$-norm is a function of $y$, and this norm is unscaled. By Lemma 9, (5.13) and (5.15) the global Schauder estimate (5.2) gives

$$
\left\|r_{k, \varepsilon}\right\|_{C_{\varepsilon}^{2, \alpha}} \leq C\left(\varepsilon^{2}+\|\varepsilon \nabla f \nabla h\|_{C_{\varepsilon}^{2, \alpha}}\right) \leq C\left(\varepsilon^{2}+\|h\|_{C_{\varepsilon}^{3, \alpha}(\Omega)}\right) .
$$

The relation between weighted and the usual Hölder norms is (up to a constant)

$$
\varepsilon^{k+\alpha}\|u\|_{C^{k, \alpha}} \leq\|u\|_{C_{\varepsilon}^{k, \alpha}} \leq \varepsilon^{k+\alpha}\|u\|_{C^{k, \alpha}}+\|u\|_{C^{0}} .
$$

Thus, we have

$$
\begin{gathered}
\left\|r_{k, \varepsilon}\right\|_{C_{\varepsilon}^{2, \alpha}} \leq C\left(\varepsilon^{2}+\varepsilon^{3+\alpha}\|h\|_{C^{3, \alpha}(\Omega)}+\|h\|_{C^{0}(\Omega)}\right) \\
\leq C\left(\varepsilon^{2}+\varepsilon^{3+\alpha}\left(\varepsilon^{2}+\left\|r_{k, \varepsilon}\right\|_{C^{2, \alpha}}\right)\right) \leq C\left(\varepsilon^{2}+\varepsilon\left\|r_{k, \varepsilon}\right\|_{C_{\varepsilon}^{2, \alpha}}\right) .
\end{gathered}
$$

Therefore, $\left\|r_{k, \varepsilon}\right\|_{C_{\varepsilon}^{2, \alpha}}=O\left(\varepsilon^{2}\right)$, which also implies that

$$
\frac{\partial^{2} w_{k, \varepsilon}}{\partial y^{2}}, \quad \frac{\partial^{2} w_{k, \varepsilon}}{\partial x_{j} \partial y}=O(\varepsilon),
$$

where $\left(x_{1}, \ldots, x_{n}\right)$ is any local coordinate system on $M$. Using the global Schauder estimate on (5.17) again, we get

$$
\frac{\partial^{2} w_{k, \varepsilon}}{\partial x_{j} \partial x_{i}}=O(\varepsilon)
$$

Lemma 11. $\left|\nabla r_{k, \varepsilon}\right|=O\left(\varepsilon^{2}\right)$.

Proof. We need to prove that for any first-order differential operator $R$ on $M$,

$$
R\left(r_{k, \varepsilon}\right)=O\left(\varepsilon^{2}\right)
$$

uniformly for any $0 \leq y \leq \varepsilon$. By Lemma 10, this is equivalent to

$$
v=R\left(r_{k, \varepsilon}(x, y f(x))\right)=O\left(\varepsilon^{2}\right)
$$

uniformly for any $0 \leq y \leq \varepsilon$.

We first assume that the vector field $R$ is vertical to $\partial M$. Then by Lemma 10, $v=0$ on $B_{\mathrm{I}}$, and

$$
v=R\left(\varepsilon \nabla f(x) \nabla w_{k, \varepsilon}(x, \varepsilon f(x))\right)=O\left(\varepsilon^{2}\right) \text { on } B_{\mathrm{II}} .
$$

On $B_{I I I}$, since $R$ is vertical to $\partial M, v=g \frac{\partial}{\partial n}$ on $\partial M$ for some function $g$. Thus by Lemma 10 again,

$$
v=g \frac{\partial}{\partial n}\left(r_{k, \varepsilon}(x, y f(x))\right)=O\left(\varepsilon^{2}\right)
$$


By (5.13) and Lemma 10, we have

$$
\tilde{\Delta} R\left(r_{k, \varepsilon}\right)+\mu_{k}(\varepsilon) R\left(r_{k, \varepsilon}\right)=R\left(F_{2}\right)+[\Delta, R] r_{k, \varepsilon}=O(1) .
$$

Thus for $C>0$ large enough, we have

$$
\tilde{\Delta}\left(R\left(r_{k, \varepsilon}\right)+C y^{2}\right)>0
$$

and by the maximum principle, $R\left(r_{k, \varepsilon}\right) \leq O\left(\varepsilon^{2}\right)$. Like in the proof of Lemma 9 , the other side of the inequality can be obtained by estimating $R\left(r_{k, \varepsilon}\right)-C y^{2}$.

Now we assume that $R$ is tangential on $\partial M$. We have similar estimates on $B_{I}$ and $B_{I I}$ as above. On $B_{I I I}$, we note that

$$
\frac{\partial}{\partial n}\left(R\left(r_{k, \varepsilon}(x, y f(x))\right)=\tilde{R}\left(r_{k, \varepsilon}(x, y f(x))\right)+O\left(\varepsilon^{2}\right),\right.
$$

where $\tilde{R}=\left[\frac{\partial}{\partial n}, R\right]$. Let $\xi$ be a function on $M$ such that on $\partial M, \xi=0 ; \partial \xi / \partial n=1$; and on $M, \varepsilon \Delta \xi$ bounded; $\xi=O(\varepsilon)$. We construct such a function $\xi$ as follows: let $\left.\rho_{(} x\right)$ be a cut-off function on $\mathbb{R}$ such that $\rho \geq 0 ; \rho^{\prime}(0)=0$ and $\rho(x)=0$ for $x \geq \varepsilon$; $\varepsilon\left|\rho_{1}\right|$ and $\varepsilon^{2}\left|\rho_{1}^{\prime \prime}\right|$ are bounded. Let $\xi=-d \rho(d)$, where $d$ is the distance function to the boundary $\partial M$. Let

$$
\tilde{v}=R\left(r_{k, \varepsilon}(x, y f(x))\right)-C_{1} \xi \max \left|\nabla r_{k, \varepsilon}\right|+C_{2} y^{2}
$$

for large $C_{1}, C_{2}$. By choosing $C_{1}$ large enough, from (5.21), we have

$$
\frac{\partial \tilde{v}}{\partial n}<0
$$

on $B_{\text {III }}$. Fixing $C_{1}$, we choose $C_{2}$ large enough. Then by Lemma 9 , we have $\tilde{\Delta} \tilde{v}>0$. By the maximum principle, the maximum point of $\tilde{v}$ must be reached on $B_{\mathrm{I}} \cup B_{\mathrm{II}}$. By the boundary conditions (5.14), we have

$$
\tilde{v} \leq C\left(\varepsilon^{2}+\varepsilon \max \left|\nabla r_{k, \varepsilon}\right|\right) \text {. }
$$

Thus we have

$$
R\left(r_{k, \varepsilon}(x, y f(x))\right) \leq C\left(\varepsilon^{2}+\varepsilon \max \left|\nabla r_{k, \varepsilon}\right|\right) .
$$

Since $R$ is arbitrary, this yields

$$
\max \left|\nabla r_{k, \varepsilon}\right| \leq C\left(\varepsilon^{2}+C_{1} \delta \max \left|\nabla r_{k, \varepsilon}\right|\right) .
$$

Proof of Theorem 5. The proof is similar to that of Lemma 10. On $B_{\mathrm{I}}$, $R\left(r_{k, \varepsilon}\right)=0$; on $B_{I I}$, by $(5.18)$, we have

$$
\left\|R\left(r_{k, \varepsilon}\right)\right\|_{C_{\varepsilon}^{2, \alpha}\left(B_{I I}\right)} \leq C \varepsilon^{2}+C \varepsilon^{3+\alpha}\|h\|_{C^{4, \alpha}(\Omega)} .
$$

On $B_{I I I}$, we have two cases. If $R$ is vertical to $\partial M$, by (5.19),

$$
\left\|R\left(r_{k, \varepsilon}\right)\right\|_{C_{\varepsilon}^{2, \alpha}\left(B_{I I I}\right)} \leq C\left(\varepsilon^{2}+\varepsilon\left\|\nabla r_{k, \varepsilon}\right\|_{\left.C_{\varepsilon}^{2, \alpha}\right)} .\right.
$$

If $R$ is tangential to $\partial M$, by (5.21),

$$
\left\|\frac{\partial}{\partial n} R\left(r_{k, \varepsilon}\right)\right\|_{C_{\varepsilon}^{1, \alpha}\left(B_{I I I}\right)} \leq C \varepsilon^{2}+\left\|\nabla r_{k, \varepsilon}\right\|_{C_{\varepsilon}^{1, \alpha}}
$$

By the Schauder estimate and (5.20),

$$
\left\|R\left(r_{k, \varepsilon}\right)\right\|_{C_{\varepsilon}^{2, \alpha}} \leq C\left(\varepsilon^{2}+\varepsilon^{3+\alpha}\|h\|_{C^{4, \alpha}(\Omega)}+\left\|\nabla r_{k, \varepsilon}\right\|_{C_{\varepsilon}^{1, \alpha}}\right) .
$$


By the Schauder interior estimate,

$$
\|h\|_{C^{4, \alpha}(\Omega)} \leq C\left(\varepsilon^{2}+\left\|r_{k, \varepsilon}\right\|_{C^{3, \alpha}}\right) \leq C\left(\varepsilon^{2}+\varepsilon^{-2-\alpha}\left\|\nabla r_{k, \varepsilon}\right\|_{C_{\varepsilon}^{2, \alpha}}\right) .
$$

Combining (5.22), (5.23) and (5.24), we get

$$
\left\|\nabla r_{k, \varepsilon}\right\|_{C_{\varepsilon}^{2, \alpha}} \leq C\left(\varepsilon^{2}+\varepsilon\left\|\nabla r_{k, \varepsilon}\right\|_{C_{\varepsilon}^{2, \alpha}}+\left\|\nabla r_{k, \varepsilon}\right\|_{C_{\varepsilon}^{1, \alpha}}\right),
$$

which implies the theorem by the interpolation inequalities.

\section{Acknowledgments}

The first author is supported by NSF grant no. DMS-09-04653. The second author gratefully acknowledges the support of the Hausdorff Center for Mathematics and the Max Planck Institut für Mathematik in Bonn. Both authors would like to thank the anonymous referee for comments which improved the quality of the paper.

\section{References}

[1] B. Andrews, Gradient and oscillation estimates and their applications in geometric PDE, AMS/IP Studies in Advanced Mathematics, 5th ICCM (2010).

[2] B. Andrews and J. Clutterbuck, Proof of the fundamental gap conjecture, 2010, arXiv 1006.1686.

[3] D. Bakry and M. Émery, Diffusions hypercontractives, Séminaire de probabilités, XIX, 1983/84, Lecture Notes in Math., vol. 1123, Springer, Berlin, 1985, 177-206 (French).

[4] D. Bakry and Z. Qian, Some new results on eigenvectors via dimension, diameter, and Ricci curvature, Adv. Math. 155(1) (2000), 98-153.

[5] I. Chavel, Eigenvalues in Riemannian geometry, Pure and Applied Mathematics, vol. 115, Academic Press Inc., Orlando, FL, 1984. Including a chapter by Burton Randol; With an appendix by Jozef Dodziuk.

[6] J. Cheeger and T. Colding, On the structure of spaces with Ricci curvature bounded below, III, J. Differ. Geom. 54(1) (2000), 37-74.

[7] E.B. Davies and B. Simon, Ultracontractivity and the heat kernel for Schrödinger operators and Dirichlet Laplacians, J. Funct. Anal. 59(2) (1984), 335-395.

[8] L. Friedlander and M. Solomyak, On the spectrum of the Dirichlet Laplacian in a narrow strip, Israel J. Math. 170 (2009), 337-354.

[9] A. Futaki and Y. Sano, Lower diameter bounds for compact shrinking Ricci solitions. arXiv: $1007.1759 \mathrm{v} 1$.

[10] D. Grieser, Thin tubes in mathematical physics, global analysis and spectral geometry, Proc. Symp. Pure Math. 77 (2008), 565-593.

[11] D. Grieser and D. Jerison, Asymptotics of eigenfunctions on plane domains, Pacific J. Math. 240(1) (2009), 109-133.

[12] D. Gilbarg and N.S. Trudinger, Elliptic partial differential equations of second order, Classics in Mathematics, Springer-Verlag, Berlin, 2001. Reprint of the 1998 edn.

[13] W. Kirsch and B. Simon, Comparison theorems for the gap of Schrödinger operators, J. Funct. Anal. 75(2) (1987), 396-410.

[14] P. Kröger, On the spectral gap for compact manifolds, J. Differ. Geom. 36(2) (1992), 315-330.

[15] P. Li and S.T. Yau, Estimates of eigenvalues of a compact Riemannian manifold, Proc. Symp. Pure Math., XXXVI, Amer. Math. Soc., Providence, RI, 1980, 205-239.

[16] J. Ling, Estimates on the lower bound of the first gap, Commun. Anal. Geom. 16(3) (2008), $539-563$.

[17] J. Ling and Z. Lu, Bounds of eigenvalues on Riemannian manifolds, Trends in partial differential equations, Adv. Lect. Math. (ALM), vol. 10, Int. Press, Somerville, MA, 2010, pp. 241-264.

[18] J. Lott, Some geometric properties of the Bakry-Émery-Ricci tensor, Comment. Math. Helv. 78(4) (2003), 865-883.

[19] L. Ma and B. Liu, Li-Yau type eigenvalue estimates for drifting Laplacians, preprint.

[20] - Convex eigenfunction of a drifting Laplacian operator and the fundamental gap, Pacific J. Math. 240(2) (2009), 343-361. 
[21] R. Schoen and S.-T. Yau, Lectures on differential geometry, Conference Proceedings and Lecture Notes in Geometry and Topology, I, International Press, Cambridge, MA, 1994.

[22] I.M. Singer, B. Wong, S.-T. Yau, and S. S.-T. Yau, An estimate of the gap of the first two eigenvalues in the Schrödinger operator, Ann. Scuola Norm. Sup. Pisa Cl. Sci. (4) 12(2) (1985), $319-333$.

[23] K. Uhlenbeck, Generic properties of eigenfunctions, Amer. J. Math. 98(4) (1976), 1059-1078.

[24] G. Wei and W. Wylie, Comparison geometry for the Bakry-Emery Ricci tensor, J. Differ. Geom. 83(2) (2009), 377-405.

[25] S.-T. Yau, Nonlinear analysis in geometry, Enseign. Math. (2) 33(1-2) (1987), 109-158.

[26] - An estimate of the gap of the first two eigenvalues in the Schrödinger operator, Lectures on partial differential equations, New Stud. Adv. Math., vol. 2, Int. Press, Somerville, MA, 2003, $223-235$.

[27] - Gap of the first two eigenvalues of the Schrödinger operator with nonconvex potential, Mat. Contemp. 35 (2008) 267-285.

[28] J.Q. Zhong, On the estimate of the first eigenvalue of a compact Riemannian manifold, Sci. Sinica Ser. A 27(12) (1984), 1265-1273.

[29] Q.H. Yu and J.Q. Zhong, Lower bounds of the gap between the first and second eigenvalues of the Schrödinger operator, Trans. Amer. Math. Soc. 294(1) (1986), 341-349.

Department of Mathematics, University of California, Irvine, USA

E-mail address: zlu@uci.edu

Max Planck Institut für Mathematik, Vivatgasse 7, Bonn, Germany.

E-mail address: rowlett@mpim-bonn.mpg.de 